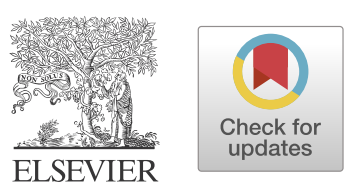

CJC Open 3 (2021) 1079-1081

Case Report

\title{
Exercise Testing With Flecainide Demonstrates Provocable Brugada Syndrome
}

\author{
Sina Safabakhsh, BSc, Andrew D. Krahn, MD, FHRS, and Zachary Laksman, MD, MSc \\ Division of Cardiology, University of British Columbia, Vancouver, British Columbia, Canada
}

\begin{abstract}
A young man with baseline early repolarization was initiated on flecainide and diltiazem for symptomatic atrial arrhythmias. A treadmill stress test induced a type 1 Brugada electrocardiogram pattern at higher heart rates. Flecainide was discontinued. Genetic testing revealed no SCN5A mutations, and a 3-generation pedigree revealed no events of concern. In this case report, we review the use-dependent properties of flecainide. We also discuss how this property can be exploited during exercise stress testing to provoke the diagnostic type 1 Brugada pattern at higher heart rates.
\end{abstract}

\section{RÉSUMÉ}

Chez un jeune homme présentant au départ une repolarisation précoce, un traitement à base de flécaïnide et de diltiazem a été instauré pour lutter contre les arythmies auriculaires symptomatiques. Une épreuve d'effort sur tapis roulant a permis d'obtenir un électrocardiogramme caractéristique d'un syndrome de Brugada de type 1 à des fréquences cardiaques plus élevées. Le traitement par le flécaiinide a été arrêté. Des analyses génétiques ont mis en évidence un résultat négatif pour les mutations du gène $S C N 5 A$, et un examen de l'arbre généalogique sur 3 générations n'a révélé aucun événement préoccupant. Dans cette étude de cas, nous analysons la propriété du flécaïnide d'avoir une efficacité proportionnelle à son utilisation. Nous évoquons également la possibilité d'exploiter cette propriété lors des épreuves d'effort afin de provoquer l'apparition du profil d'ECG du syndrome de Brugada de type 1 à des fréquences cardiaques plus élevées.

\section{Novel Teaching Points}

- Flecainide use-dependence allows for provocation of the diagnostic type 1 Brugada pattern at higher heart rates.

- Flecainide challenge during an exercise stress test can aid in $\mathrm{BrS}$ diagnosis by unmasking the diagnostic type 1 pattern at higher heart rates.

related to Brugada syndrome $(\mathrm{BrS})$ in the context of a baseline ECG with early repolarization in the anterior precordial leads, an exercise treadmill stress test was organized. The patient developed a type 1 Brugada ECG pattern on treadmill exercise stress testing, which was most evident at high heart rates and resolved in recovery (Fig. 2; Supplemental Fig. S2; Supplemental Tables S1 and S2). The patient was asked to discontinue flecainide and meet with a genetic counsellor for SCN5A testing and a detailed family history. The patient was advised to avoid medications on the Brugada "medications to avoid" list (BrugadaDrugs.org).

There were no SCN5A mutations upon genetic testing. A 3generation pedigree revealed no immediate or extended family history or events of concern. Cascade family screening was initiated. High lead ECG after flecainide discontinuation did not show evidence of BrS in the proband (Supplemental Fig. S1). 


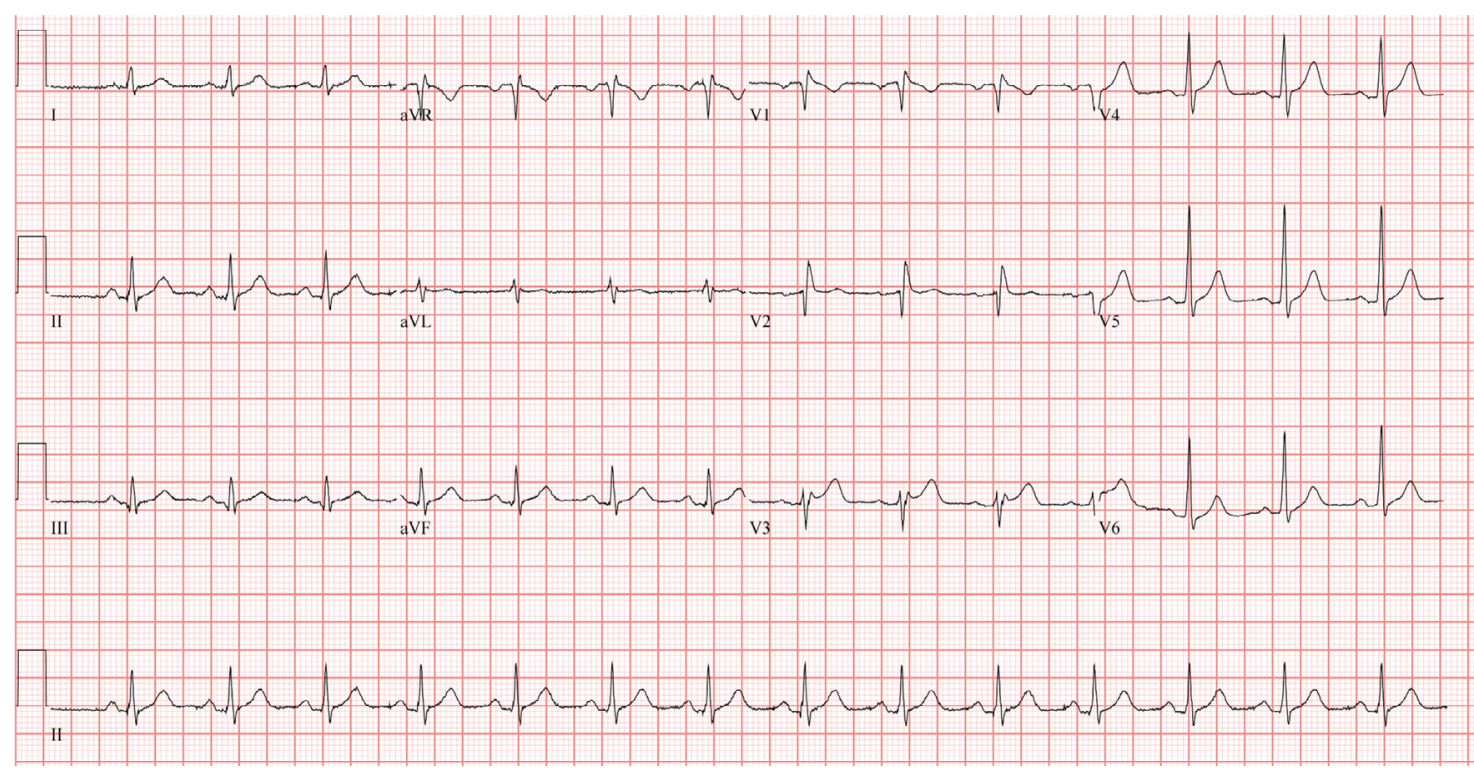

Figure 1. Baseline supine electrocardiogram prior to flecainide therapy onset, suspicious for type 2 Brugada pattern in $V_{3}$.

$\mathbf{A}$

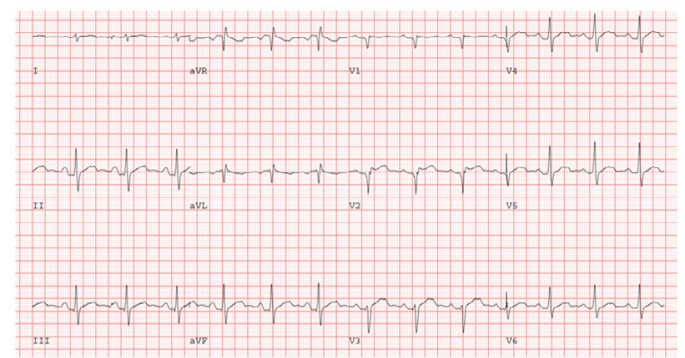

C

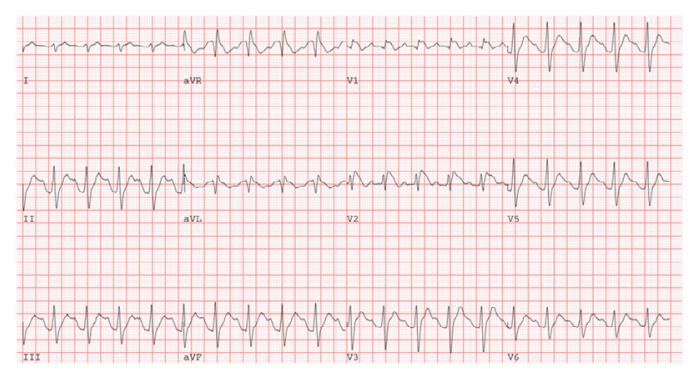

B

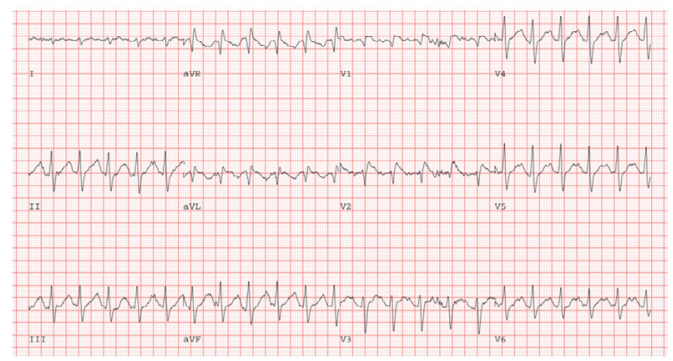

D

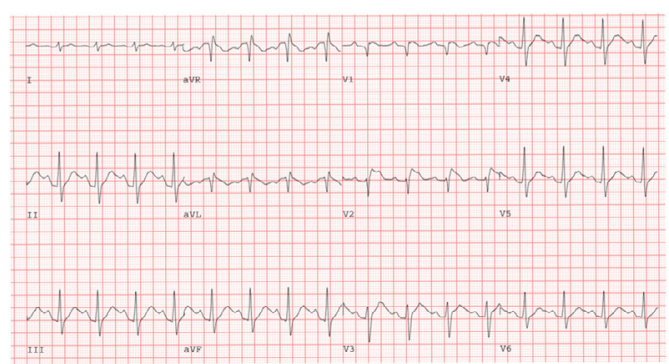

Figure 2. Exercise treadmill stress test electrocardiograms showing development of type 1 Brugada pattern at higher heart rates: (A) pretest; (B) running at $3.4 \mathrm{mph}$ (Stage 3 Bruce protocol); (C) 1 minute, 5 seconds of recovery; and (D) 3 minutes, 5 seconds of recovery. Incomplete resolution of the type 1 pattern is noted at the end of recovery.

\section{Discussion}

$\mathrm{BrS}$ is characterized by an ECG finding of coved ST-segment elevation followed by a negative $\mathrm{T}$-wave in the right precordial leads. ${ }^{1}$ Although some patients present with syncope or sudden cardiac arrest, most will be asymptomatic. Therefore, an important diagnostic tool is a challenge study with a sodium-channel blocker to unmask the diagnostic type 1 ECG pattern.

Flecainide is 1 of 3 sodium-channel blockers used to perform a challenge study. It displays use-dependence whereby it blocks voltage-gated sodium channels at higher rates of depolarization. ${ }^{2,3}$ This display makes flecainide especially effective at unmasking the Brugada phenotype at higher heart rates, such as during exercise. Clinically, this unmasking could allow for the development of exercise stress test protocols that use flecainide to identify provocable BrS. Clinicians should carefully evaluate for early repolarization before initiating flecainide, as this medication may have tragic consequences in patients with undiagnosed $\mathrm{BrS}$.

The pathophysiology of BrS explains the efficacy of sodiumchannel blockade in unmasking characteristic ECG patterns. According to the repolarization theory, the characteristic ST- 
segment elevation in $\mathrm{BrS}$ results from a transmural voltage gradient between the endo- and epicardium of the right ventricular outflow tract. ${ }^{1}$ This gradient is due to an enhanced $\mathrm{I}_{\text {to }}$-mediated action potential notch and loss of action potential dome in the epicardium. This loss can result from any underlying mechanism that decreases inward current or increases outward current. Sodium-channel blockers temporarily reduce inward sodium current to unmask BrS ECG patterns. Furthermore, enhanced sodium-channel inactivation is one mechanism of reduced inward current in inherited BrS. ${ }^{4}$ At higher body temperatures, such as during exercise stress testing, inactivation is further accentuated due to channel-gating kinetics. ${ }^{1}$ As flecainide blockade normally increases after sodium-channel inactivation, this process becomes enhanced in this case to effectively unmask BrS ECG patterns. It has also been shown that calcium-channel blockade can contribute to unmasking of $\mathrm{BrS}$ ECG patterns by reducing inward calcium current. ${ }^{3}$ In this case, it is possible that diltiazem may have further contributed to unmasking the BrS ECG pattern.

\section{Funding Sources}

A University of British Columbia Cardiology Academic Practice Plan and a Michael Smith Foundation for Health Research (MSFHR) Health Professional Investigator Award to $\mathrm{ZL}$ provided funding for this work. The authors have no other funding sources to declare.

\section{Disclosures}

The authors have no conflicts of interest to disclose.

\section{References}

1. Brugada J, Campuzano O, Arbelo E, Sarquella-Brugada G, Brugada R. Present status of Brugada syndrome. J Am Coll Cardiol 2018;72:1046-59.

2. Ranger S, Talajic M, Lemery R, Roy D, Nattel S. Amplification of flecainide-induced ventricular conduction slowing by exercise. A potentially significant clinical consequence of use-dependent sodium channel blockade. Circulation 1989;79:1000-6.

3. Salvage SC, Chandrasekharan KH, Jeevaratnam K, et al. Multiple targets for flecainide action: implications for cardiac arrhythmogenesis. Br J Pharmacol 2018;175:1260-78.

4. Wang DW, Makita N, Kitabatake A, Balser JR, George AL. Enhanced Na (+) channel intermediate inactivation in Brugada syndrome. Circ Res 2000;87:E37-43.

5. Fish JM, Antzelevitch C. Role of sodium and calcium channel block in unmasking the Brugada syndrome. Heart Rhythm 2004;1:210-7.

\section{Supplementary Material}

To access the supplementary material accompanying this article, visit CJC Open at https://www.cjcopen.cal and at doi:10.1016/j.cjco.2021.04.001. 